PROCEEDINGS OF THE

AMERICAN MATHEMATICAL SOCIETY

Volume 138, Number 9, September 2010, Pages 3285-3291

S 0002-9939(10)10463-8

Article electronically published on April 30, 2010

\title{
NON-REPRESENTABILITY OF FINITE PROJECTIVE PLANES BY CONVEX SETS
}

\author{
MARTIN TANCER
}

(Communicated by Jonathan I. Hall)

\begin{abstract}
We prove that there is no $d$ such that all finite projective planes can be represented by convex sets in $\mathbb{R}^{d}$, answering a question of Alon, Kalai, Matoušek, and Meshulam. Here, if $\mathbb{P}$ is a projective plane with lines $\ell_{1}, \ldots, \ell_{n}$, a representation of $\mathbb{P}$ by convex sets in $\mathbb{R}^{d}$ is a collection of convex sets $C_{1}, \ldots, C_{n} \subseteq \mathbb{R}^{d}$ such that $C_{i_{1}}, C_{i_{2}}, \ldots, C_{i_{k}}$ have a common point if and only if the corresponding lines $\ell_{i_{1}}, \ldots, \ell_{i_{k}}$ have a common point in $\mathbb{P}$. The proof combines a positive-fraction selection lemma of Pach with a result of Alon on "expansion" of finite projective planes. As a corollary, we show that for every $d$ there are 2-collapsible simplicial complexes that are not $d$-representable, strengthening a result of Matoušek and the author.
\end{abstract}

\section{INTRODUCTION}

Intersection patterns of convex sets. One of the important areas in discrete geometry regards understanding "intersection patterns" of convex sets. A pioneering result in this area is Helly's theorem [8] which asserts that if $C_{1}, C_{2}, \ldots, C_{n}$ are convex sets in $\mathbb{R}^{d}, n \geq d+1$ and every $d+1$ of the $C_{i}$ have a common point, then $\bigcap_{i=1}^{n} C_{i} \neq \emptyset$.

Consequently, intersection patterns of convex sets have been studied intensively. This study led to the introduction of $d$-representable and $d$-collapsible simplicial complexes. We recall that the nerve of a family $\mathcal{S}=\left\{S_{1}, S_{2}, \ldots, S_{n}\right\}$ is the simplicial complex with vertex set $[n]:=\{1,2, \ldots, n\}$ and with a set $\sigma \subseteq[n]$ forming a simplex if $\bigcap_{i \in \sigma} S_{i} \neq \emptyset$. A simplicial complex $\mathrm{K}$ is $d$-representable if it is isomorphic to the nerve of a family of convex sets in $\mathbb{R}^{d}$. In this language Helly's theorem implies that a $d$-representable complex is determined by its $d$-skeleton. We refer to [5, 6, 10, 12, for more examples and background.

Wegner in his seminal 1975 paper [15] introduced $d$-collapsible simplicial complexes. To define this notion, we first introduce an elementary $d$-collapse. Let $\mathrm{K}$ be a simplicial complex and let $\sigma, \tau \in \mathrm{K}$ be faces (simplices) such that

(i) $\operatorname{dim} \sigma \leq d-1$,

(ii) $\tau$ is an inclusion-maximal face of $\mathrm{K}$,

Received by the editors August 28, 2009.

2010 Mathematics Subject Classification. Primary 52A35, 52A20; Secondary 05B25, 05E45.

Key words and phrases. Convex set, intersection pattern, $d$-representable, $d$-collapsible, finite projective plane.

The author was partially supported by project GAUK 49209. He was also supported by project 1M0545 of The Ministry of Education of the Czech Republic.

(C) 2010 American Mathematical Society
Reverts to public domain 28 years from publication 


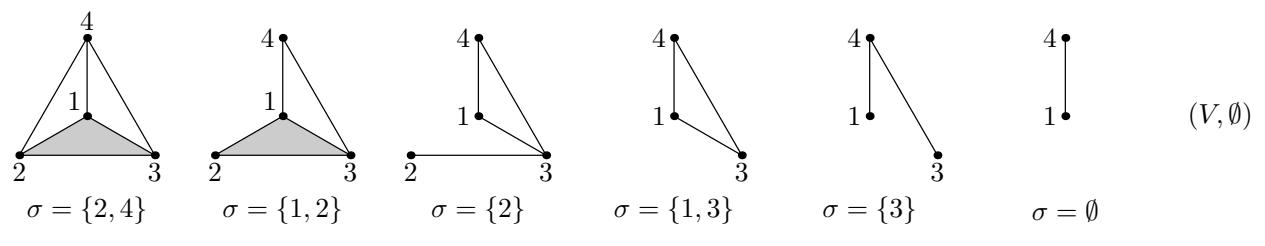

Figure 1. An example of 2-collapsing.

(iii) $\sigma \subseteq \tau$, and

(iv) $\tau$ is the only face of $\mathbf{K}$ satisfying (ii) and (iii).

Then we say that $\sigma$ is a $d$-collapsible face of $\mathrm{K}$ and that the simplicial complex $\mathrm{K}^{\prime}:=$ $\mathrm{K} \backslash\{\eta \in \mathrm{K}: \sigma \subseteq \eta \subseteq \tau\}$ arises from $\mathrm{K}$ by an elementary $d$-collapse. A simplicial complex $\mathrm{K}$ is $d$-collapsible if there exists a sequence of elementary $d$-collapses that reduces $\mathrm{K}$ to the empty complex $\emptyset$. Figure 1 shows an example of 2-collapsing.

Wegner [15] showed that every $d$-representable simplicial complex is $d$-collapsible and also gave an example of a 2-collapsible simplicial complex that is not 2-representable.

Alon et al. [4, Problem 17] asked whether there is a function $f=f(d)$ such that every $d$-collapsible simplicial complex is $f(d)$-representable. Matoušek and Tancer [12] showed that $f(d) \geq 2 d-1$ if it exists. We improve this result by showing that no such $f$ exists.

Finite projective planes. A finite projective plane of order $q \geq 2$ is a pair $(P, \mathcal{L})$ where $P$ is a finite set of points, and $\mathcal{L} \subseteq 2^{P}$ is a set of subsets of $P$ (called lines) such that (i) every two points are contained in a unique line, (ii) every two lines intersect in a unique point, and (iii) every line contains $q+1$ points. It follows that every point is contained in $q+1$ lines and $|P|=|\mathcal{L}|=q^{2}+q+1$; see e.g. [11] for more details.

It is well known that a projective plane of order $q$ exists whenever $q$ is a power of a prime. We remark that it is a well known open problem to decide whether there are projective planes of other orders.

It is also known that a finite projective plane cannot be represented in $\mathbb{R}^{d}$ so that the points of the projective plane are points in $\mathbb{R}^{d}$ and the lines of the projective plane are the inclusionwise maximal collections of points lying on a common Euclidean line. This fact follows for example from the Sylvester-Gallai theorem: if $p_{1}, \ldots, p_{n}$ are points in the plane not all of them lying on a common line, then there is a line in the plane which intersects exactly two of these points (see 7 for the original solution and 9 for an elegant proof). Our task is to obtain a similar result where the Euclidean lines are replaced by convex sets.

Let $(X, \mathcal{F})$ be a set system where $X$ is a finite set and $\mathcal{F}$ is a set of some subsets of $X$. We say that $(X, \mathcal{F})$ is representable by convex sets in $\mathbb{R}^{d}$ if there are convex sets $C_{F} \subset \mathbb{R}^{d}$ for $F \in \mathcal{F}$ such that for any $F_{1}, \ldots, F_{k} \in \mathcal{F}$ the convex sets $C_{F_{1}}, \ldots, C_{F_{k}}$ intersect if and only if the sets $F_{1}, \ldots, F_{k}$ have a common point in $X \mathbb{1}$

\footnotetext{
${ }^{1}$ We strongly distinguish the terms $d$-representable simplicial complex and a set system representable by convex sets in $\mathbb{R}^{d}$; they have a different meaning. In fact, they are dual in a certain sense. A set system $(X, \mathcal{F})$ is representable by convex sets in $\mathbb{R}^{d}$ if and only if the nerve of $\mathcal{F}$ is $d$-representable.
} 


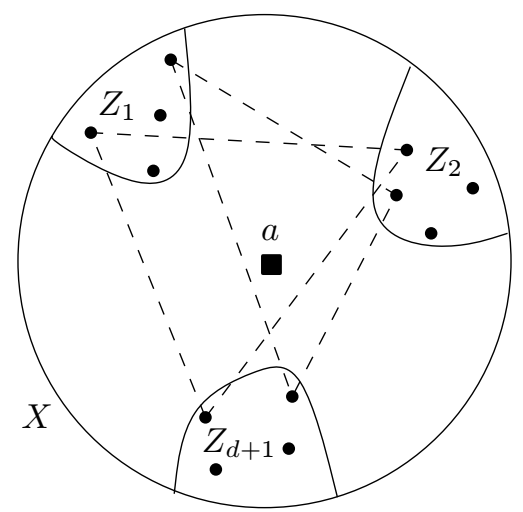

Figure 2. Positive-fraction selection lemma: every triangle formed by the sets $Z_{i}$ contains $a$.

Theorem 1.1. For every $d \in \mathbb{N}$ there is a $q_{0}=q_{0}(d) \in \mathbb{N}$ such that any projective plane $(P, \mathcal{L})$ of order $q \geq q_{0}$ is not representable by convex sets in $\mathbb{R}^{d}$.

We remark that the assumption $q \geq q_{0}$ cannot be left out since every projective plane $(P, \mathcal{L})$ of order $q$ can be easily represented by convex sets in $\mathbb{R}^{q^{2}+q}$. For consider $P$ as the set of vertices of a $\left(q^{2}+q\right)$-simplex in $\mathbb{R}^{q^{2}+q}$ and set $C_{\ell}:=$ $\operatorname{conv}\{p: p \in \ell\}$ for a line $\ell \in \mathcal{L} 2$

Our main tools are the positive-fraction selection lemma and the fact that the projective planes (considered as bipartite graphs) are expanders.

Theorem 1.1 is proved in Section 2 .

We also have the following consequence of Theorem 1.1, which answers the question of Alon at al. 4] (as announced above) and which is proved in Section 3 .

Corollary 1.2. Let $d>1$ be an integer and let $q_{0}=q_{0}(d)$ be the integer from Theorem 1.1. Let $(P, \mathcal{L})$ be the projective plane of order $q \geq q_{0}$. Let $\mathrm{K}_{q}$ be a simplicial complex whose vertices are points in $P$ and whose faces are subsets of lines in $\mathcal{L}$. Then $\mathrm{K}_{q}$ is 2-collapsible and is not d-representable.

\section{Proof of the main Result}

In this section we prove Theorem 1.1 We need a few preliminaries. Assume that $\left(Z_{1}, \ldots, Z_{k}\right)$ is a $k$-tuple of sets. By a transversal of this $k$-tuple we mean any set $T=\left\{t_{1}, \ldots, t_{k}\right\}$ such that $t_{i} \in Z_{i}$ for every $i \in[k]$. We need the following result due to Pach [13; see also [10, Theorem 9.5.1]. See Figure 2,

Theorem 2.1 (Positive-fraction selection lemma; a special case). For every natural number $d$, there exists $c=c(d)>0$ with the following property. Let $X \subset \mathbb{R}^{d}$ be a finite set of points in general position (i.e., there are no $d+1$ points lying in a common hyperplane). Then there is a point $a \in \mathbb{R}^{d}$ and disjoint subsets $Z_{1}, \ldots, Z_{d+1}$, with $\left|Z_{i}\right| \geq c|X|$ such that the convex hull of every transversal of $\left(Z_{1}, \ldots, Z_{d+1}\right)$ contains a.

\footnotetext{
${ }^{2}$ With a bit more effort it can be shown that a projective plane of order $q$ can be represented by convex sets in $\mathbb{R}^{2 q+1}$. This is based on the fact that a simplicial complex of dimension $d$ is $(2 d+1)$-representable.
} 
We remark that the proof of Theorem 2.1 uses several involved tools such as the weak hypergraph regularity lemma or the same-type lemma (therefore we do not reproduce any details of the proof here). We should also remark that this is only a special case of Pach's theorem (but general enough); Pach moreover assumes that $Z_{i} \subseteq X_{i}$, where $X_{1} \cup \cdots \cup X_{d+1}$ is a partition of $X$, and in this setting $\left|Z_{i}\right| \geq c\left|X_{i}\right|$.

We also need the following expansion property of the projective plane [1, Theorem 2.1], 2].

Theorem 2.2. Let $(P, \mathcal{L})$ be a projective plane of order $q$. Let $A \subseteq P$. Then $|\{\ell \in \mathcal{L}: \ell \cap A=\emptyset\}| \leq n^{3 / 2} /|A|$, where $n=q^{2}+q+1$.

Alon, Haussler and Welzl [3] used this expansion property in the context of range-searching problems. They showed that the points of a projective plane (of high enough order) cannot be partitioned into a small number of sets $P_{1}, \ldots$, $P_{m}$ so that for every projective line $\ell$ the set $\bigcup_{\ell \cap P_{i} \neq \emptyset, P_{i}} P_{i}$ contains only a given fraction of all the points. Known results on range-searching problems imply that a projective plane of a high order cannot be represented by halfspaces or simplices in $\mathbb{R}^{d}$. However, the author is not aware that this approach would imply the result for convex sets.

For completeness, we also reproduce a short proof of Theorem 2.2

Proof of Theorem 2.2. Let $M=\left(m_{p \ell}\right)$ be an $n \times n$ matrix with rows indexed by the points of $P$ and columns indexed by the lines of $\mathcal{L}$. We set $m_{p \ell}:=1$ if $p \in \ell$ and $m_{p \ell}:=0$ otherwise. The matrix $M M^{T}$ has real nonnegative eigenvalues $\lambda_{1} \geq$ $\lambda_{2} \geq \cdots \geq \lambda_{n}$.

By a theorem of Tanner [14,

$$
|N(A)| \geq \frac{(q+1)^{2}|A|}{\left((q+1)^{2}-\lambda_{2}\right)|A| / n+\lambda_{2}},
$$

where $N(A)$ denotes $\{\ell \in \mathcal{L}: \ell \cap A \neq \emptyset\}$, the neighborhood of $A$.

It is not hard to compute that $\lambda_{1}=(q+1)^{2}$ and $\lambda_{2}=\cdots=\lambda_{n}=q$. Consequently,

$$
|N(A)| \geq \frac{(q+1)^{2}|A|}{|A|+q}=n-\frac{q(n-|A|)}{|A|+q} \geq n-\frac{n^{3 / 2}}{|A|} .
$$

Proof of Theorem 1.1. For a contradiction, we assume that $(P, \mathcal{L})$ is representable by convex sets in $\mathbb{R}^{d}$; i.e., there are convex sets $C_{\ell}$ for $\ell \in \mathcal{L}$ such that $C_{\ell_{1}}, \ldots, C_{\ell_{k}}$ intersect if and only if $\ell_{1}, \ldots, \ell_{k}$ contain a common point. By standard tricks, we can assume that these sets are open. We explain this step at the end of the proof.

Let $p \in P$. We know that $\bigcap_{p \in \ell} C_{\ell}$ is nonempty (and open). Let $x_{p}$ be a point of this intersection. We define $X:=\left\{x_{p}: p \in P\right\}$. Because of the openness of the intersections we can assume that $X$ is in general position.

Let $c=c(d)>0, a \in \mathbb{R}^{d}$, and $Z_{1}, \ldots, Z_{d+1}$ be the (output) data from Theorem 2.1 (when applied to $X$ ). We know that $\left|Z_{i}\right| \geq c|X|$. Let us set $P_{i}:=\{p \in P$ : $\left.x_{p} \in Z_{i}\right\}$ and $\mathcal{M}_{i}:=\left\{\ell \in \mathcal{L}: \ell \cap P_{i}=\emptyset\right\}$. By Theorem 2.2 with $A=P_{i}$ we get

$$
\left|\mathcal{M}_{i}\right| \leq \frac{n^{3 / 2}}{\left|P_{i}\right|}=\frac{n^{3 / 2}}{\left|Z_{i}\right|} \leq \frac{n^{3 / 2}}{c|X|}=\frac{n^{3 / 2}}{c n} \leq \frac{n}{2(d+1)}=\frac{|\mathcal{L}|}{2(d+1)}
$$

provided that $q$ (and hence $n$ as well) is sufficiently large (depending on $d$ and $c$ ). 


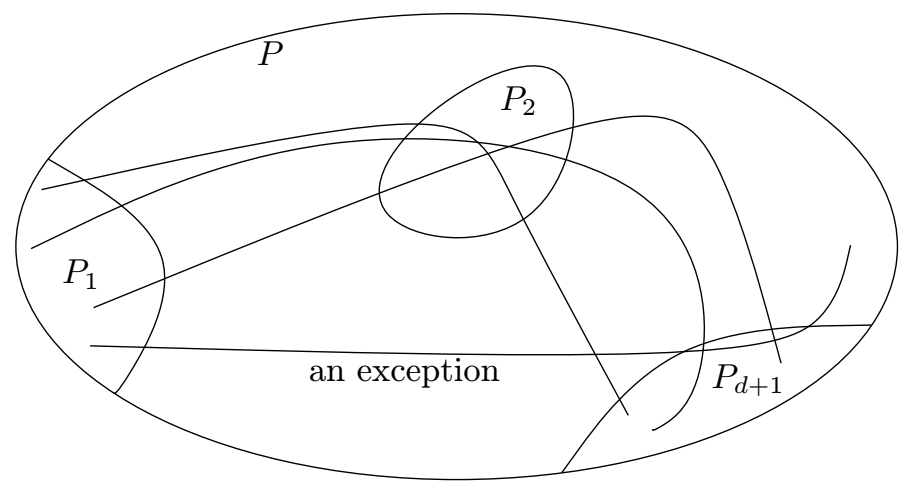

Figure 3. Almost all lines intersect all of $P_{1}, \ldots, P_{d+1}$.

Hence the set $\mathcal{L}^{\prime}:=\mathcal{L} \backslash\left(\mathcal{M}_{1} \cup \cdots \cup \mathcal{M}_{d+1}\right)$ of lines that intersect each of $P_{1}, \ldots, P_{d+1}$ contains at least half of the lines of $\mathcal{L}$; see Figure 3. Now let $\ell \in \mathcal{L}^{\prime}$ and let $p_{i} \in \ell \cap P_{i}$. Then $\left(x_{p_{1}}, \ldots, x_{p_{d+1}}\right)$ is a transversal of $\left(Z_{1}, \ldots, Z_{d+1}\right)$. Thus $a \in \operatorname{conv}\left\{x_{p_{1}}, \ldots, x_{p_{d+1}}\right\} \subseteq C_{\ell}$, and so $a$ is contained in at least $\frac{|\mathcal{L}|}{2}$ of the $C_{\ell}$. This is a contradiction since at most $q+1$ sets among the $C_{\ell}$ can have a nonempty intersection.

It remains to show that we can assume the $C_{\ell}$ open. Let us have a set of lines $\mathcal{S}=\left\{\ell_{1}, \ldots, \ell_{k}\right\}$ such that $C(\mathcal{S}):=\bigcap_{i=1}^{k} C_{\ell_{i}}$ is nonempty. In such a case we pick a point $y_{\mathcal{S}} \in C(\mathcal{S})$. For $\ell \in \mathcal{L}$ we set

$$
C_{\ell}^{\prime}:=\operatorname{conv}\left\{y_{\mathcal{S}}: \mathcal{S} \subseteq \mathcal{L}, \ell \in \mathcal{S}, C(\mathcal{S}) \neq \emptyset\right\} .
$$

In the definition of $C_{\ell}^{\prime}$ we have considered all the possible intersections; thus the sets $\left\{C_{\ell}^{\prime}: \ell \in \mathcal{L}\right\}$ have the same "intersection pattern" as the sets $\left\{C_{\ell}: \ell \in \mathcal{L}\right\}$. This means that the sets $\left\{C_{\ell}^{\prime}: \ell \in \mathcal{L}\right\}$ are a counterexample for the theorem if and only if the sets $\left\{C_{\ell}: \ell \in \mathcal{L}\right\}$ are a counterexample. The sets $C_{\ell}^{\prime}$ are compact sets means that from now on we can assume that all the considered sets are compact.

Finally we blow up these sets by a small $\varepsilon>0$. Thus we get open sets instead of compact sets.

\section{Proof of the gap Between $d$-Representability AND $d$-COLLAPSIBILITY}

Proof of Corollary 1.2. The fact that the complex $\mathrm{K}_{q}$ is 2-collapsible is essentially mentioned in [4, discussion below Problem 15] (without a proof).

All the inclusionwise maximal faces of $\mathrm{K}_{q}$ are of the form $\sigma_{\ell}=\{p: p \in \ell\}$ for $\ell \in \mathcal{L}$. Two such faces intersect only in a vertex; thus it is possible to 2-collapse these faces gradually to the vertices; the details are given in Lemma 3.1 below. After these collapsings, it is sufficient to remove the vertices (which are already inclusionwise maximal).

It remains to show that $\mathrm{K}_{q}$ is not $d$-representable. We consider the dual projective plane $(\mathcal{L}, \bar{P})$, where $\bar{P}:=\{\{\ell \in \mathcal{L}: p \in \ell\}: p \in P\}$. In particular we can identify a point $p \in P$ with a dual line $\{\ell \in \mathcal{L}: p \in \ell\} \in \bar{P}$. Theorem 1.1 applied for this dual plane $(\mathcal{L}, \bar{P})$ essentially states that $\mathrm{K}_{q}$ is not $d$-representable (convex sets in 


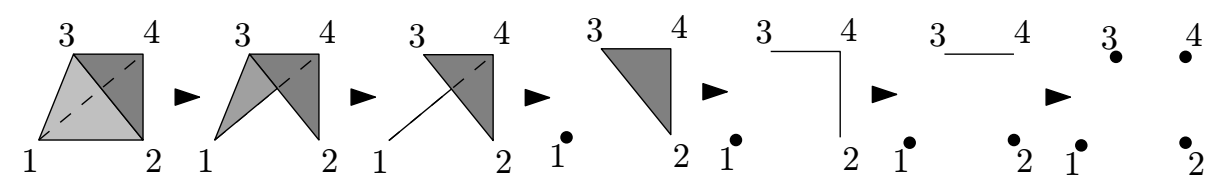

Figure 4. Collapsing a simplex to its vertices.

the statement now correspond to the lines in $\bar{P}$, which we have identified with $P$, the set of vertices of $K_{q}$ ).

Lemma 3.1. Let $\Delta$ be a d-simplex, i.e., a simplicial complex with $[d+1]$ as the set of vertices and with all possible faces. Then there is a sequence of elementary 2 -collapses that starts with $\Delta$ and ends with the simplicial complex that contains all the vertices of $\Delta$ and no faces of higher dimension.

Proof. In every elementary 2-collapse we only mention the smaller face $\sigma$ (here we adopt the notation from the definition of an elementary $d$-collapse), since a 2-collapse is uniquely determined by $\sigma$.

The following sequence of choices of $\sigma$ provides the required 2-collapsing (the faces are ordered in the lexicographical order; see Figure 4):

$$
\{1,2\},\{1,3\}, \ldots,\{1, d+1\},\{2,3\}, \ldots,\{2, d+1\},\{3,1\}, \ldots,\{d, d+1\} .
$$

\section{ACKNOWLEDGEMENT}

I would like to thank Jiří Matoušek for informing me about the result on the expansion property of projective planes and also for suggesting many improvements while reading the preliminary version of the article.

\section{REFERENCES}

1. N. Alon, Expanders, sorting in rounds and superconcentrators of limited depth, Proc. 17th ACM Sympos. on Theory of Comput., 1985, pp. 98-102.

2. N. Alon, Eigenvalues, geometric expanders, sorting in rounds, and Ramsey theory, Combinatorica 6 (1986), no. 3, 207-219. MR875289 (88g:05090)

3. N. Alon, D. Haussler, and E. Welzl, Partitioning and geometric embedding of range spaces of finite Vapnik-Chervonenkis dimension, Proc. 3rd ACM Sympos. on Computational Geometry, 1987, pp. 331-340.

4. N. Alon, G. Kalai, J. Matoušek, and R. Meshulam, Transversal numbers for hypergraphs arising in geometry, Adv. in Appl. Math. 130 (2002), 2509-2514. MR1921545 (2003g:52004)

5. L. Danzer, B. Grünbaum, and V. Klee, Helly's theorem and its relatives, Proc. Sympos. Pure Math., vol. 7 (Convexity), American Mathematical Society, Providence, RI, 1963, pp. 101-180. MR0157289 (28:524)

6. J. Eckhoff, Helly, Radon and Carathéodory type theorems, Handbook of Convex Geometry (P. M. Gruber and J. M. Wills, eds.), North-Holland, Amsterdam, 1993, pp. 389-448. MR.1242986 (94k:52010)

7. T. Gallai, Solution of problem 4065, Amer. Math. Monthly 51 (1944), 169-171. MR.1525919

8. E. Helly, Über mengen konvexer Körper mit gemeinschaftlichen Punkten, Jahresber. Deustch. Math.-Verein. 32 (1923), 175-176.

9. L.M. Kelly, A resolution of the Sylvester-Gallai problem of J.-P. Serre, Discrete Comput. Geom. 1 (1986), no. 1, 101-104. MR834051 (87k:14047)

10. J. Matoušek, Lectures on discrete geometry, Springer-Verlag New York, Inc., 2002. MR 1899299 (2003f:52011)

11. J. Matoušek and J. Nešetřil, Invitation to discrete mathematics, Oxford University Press, Oxford, 1998. MR 1668997 
12. J. Matoušek and M. Tancer, Dimension gaps between representability and collapsibility, Discrete Comput. Geom. 42 (2009), no. 4, 631-639. MR2556459

13. J. Pach, A Tverberg-type result on multicolored simplices, Comput. Geom. 10 (1998), 71-76. MR.1614605 (99a:52006)

14. R. M. Tanner, Explicit concentrators from generalized n-gons, SIAM J. Algebraic Discrete Methods 5 (1984), no. 3, 287-293. MR752035 (85k:68080)

15. G. Wegner, d-collapsing and nerves of families of convex sets, Arch. Math. 26 (1975), 317321. MR0375333 (51:11528)

Department of Applied Mathematics and Institute for Theoretical Computer Science, Faculty of Mathematics and Physics, Charles University, Malostranské nám. 25, 11800 Prague, Czech Republic

E-mail address: tancer@kam.mff.cuni.cz 